\title{
Flow-Induced Dilation in Newborn Intestine
}

\author{
PHILIP T. NOWICKI AND CHARLES E. MILLER \\ Department of Pediatrics, The Ohio State University and The Wexner Institute for Pediatric Research, \\ Childrens Hospital, Columbus, Ohio 433205
}

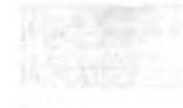

$x^{2}$

The goal of these experiments was to determine the presence and mechanistic basis of flow-induced dilation in mesenteric artery from 3-d-old swine. In the first experiment, in vitro gut loops were perfused from a blood-filled reservoir under controlled-flow conditions, and flow was progressively increased from $\sim 40 \%$ to $\sim 170 \%$ in six increments by manipulation of pump speed. Under control conditions, vascular resistance significantly decreased after each step increase in flow rate. $N^{\mathrm{G}}$ Monomethyl-L-arginine (LNMMA; $10^{-4} \mathrm{M}$ ), an arginine analog that blocks nitric oxide production, eliminated this flow-induced dilation, but only for the step increases in flow at rates above the baseline flow rate. For step increases below the baseline rate, LNMMA caused a simple parallel shift of the resistance-flow rate curve upward. Phenylephrine $\left(10^{-6} \mathrm{M}\right)$, an $\alpha_{1}$-agonist which has no effect on nitric oxide production or half-life, did not eliminate flow-induced dilation, but instead caused a simple parallel shift of the resistance-flow rate curve upward across the entire range of flows studied. In the second experiment, a $3-\mathrm{cm}$ segment of mesenteric artery was perfused with Krebs buffer at two flow rates: 10 and $25 \mathrm{~mL} / \mathrm{min}$. The effluent from the mes-

Flow-induced dilation is a vascular phenomenon wherein augmentation of flow rate through an artery results in vasodilation of that vessel (1). The process was initially described in large conduit arteries and was presumed to be part of series of vascular adjustments which facilitate oxygen delivery during exercise $(1,2)$. In this scenario, resistance vessels within the microcirculation of contracting muscle dilate in response to a metabolic feedback signal, an action which decreases downstream vascular resistance. This hemodynamic change increases flow rate through the conduit vessels leading to the muscle. The stimulus of increased flow rate induces dilation of the conduit vessels, an action which maximizes vascular conductance. Subsequent studies have identified flow-induced dilation within arterioles (3) and have established that flowinduced dilation is an endothelium-dependent process, that is,

Received March 21, 1995; accepted June 19, 1995.

Correspondence and reprint requests: Philip T. Nowicki, Childrens Hospital, 700 Childrens Drive, Columbus, $\mathrm{OH} 43205$.

Funded by award HD 25256 from the National Institute of Child Health and Human Development. enteric artery segment was suffused onto a deendothelialized, phenylephrine-precontracted ring of swine carotid artery; relaxation of this bioassay vessel served as an index of release of relaxing factors from the mesenteric artery segment. Under control conditions, increase in the mesenteric artery flow rate caused a $60 \%$ relaxation of the bioassay vessel. This effect was eliminated by the addition of LNMMA to the buffer $\left(10^{-4} \mathrm{M}\right)$, but not by the addition of indomethacin $\left(10^{-5} \mathrm{M}\right)$. Flow-induced dilation occurs in the mesenteric artery of 3 -d-old swine. This vascular phenomenon appears to be mediated by nitric oxide, but only at flows above the baseline flow rate. The mechanism(s) responsible for this phenomenon at lower flow rates is not clear. (Pediatr Res 38: 783-791, 1995)

NO, nitric oxide

\section{Abbreviations}

LNMMA, $N^{\mathrm{G}}$-monomethyl-L-arginine

(a-v) $\mathrm{O}_{2}$, arteriovenous oxygen content difference across the intestine

ANOVA, analysis of variance

a process mediated by relaxing factors produced and released by the vascular endothelium (4), especially NO (5). Others have suggested that the actual stimulus for flow-induced dilation is wall shear stress, not flow rate per se $(4,6)$. In this paradigm, augmentation of flow rate increases wall shear stress, a mechanostimulus for NO production; subsequent flow-induced dilation increases the vessel radius and restores shear stress to baseline.

To our knowledge, the phenomenon of flow-induced dilation has not been described in newborn blood vessels, although there are at least two reasons to anticipate that the process might participate in regulation of the newborn intestinal circulation. First, the endothelium-derived relaxing factor proposed as the principal mediator of flow-induced dilation, NO, is present within the newborn intestinal circulation and participates in setting its basal vascular tone (7). Second, the resting flow rate within the newborn intestinal circulation is high when compared with that noted during later life, whereas the vascular dimensions are obviously smaller (8); these circumstances predict that wall shear stress will be substantial in newborn intestine $(4,6)$. 
The goal of this investigation was to determine whether flow-induced dilation occurs in newborn intestine. To this end, in vitro gut loops from 3 -d-old swine were perfused from a blood-filled reservoir under controlled-flow conditions and the effects of stepwise manipulation of flow rate on vascular resistance and tissue oxygen uptake noted. This protocol was conducted during infusion of LNMMA or phenylephrine to determine the roles of $\mathrm{NO}$ and basal vascular tone, respectively, on flow-induced dilation. An additional protocol was carried out to determine whether flow-induced dilation is mediated by a transmissible factor released by the mesenteric artery. To this end, buffer-perfused mesenteric artery segments were mounted so that their effluent was suffused onto a deendothelialized carotid artery ring. Relaxation of the ring in response to increased perfusion rate through the mesenteric artery was accepted as evidence of flow-induced production and release of a relaxing factor. This protocol was carried out in the presence of indomethacin or LNMMA to characterize the identity of the relaxing factor.

\section{METHODS}

\section{Animal Acquisition and Handling}

Animals were obtained from a local swine farm on the day before use. The age range was $1-3 \mathrm{~d}$ old. Animals were fasted for $12 \mathrm{~h}$ before induction of anesthesia with pentobarbital sodium, $35 \mathrm{mg} / \mathrm{kg}$ intraperitoneally. Euthanasia was carried out by i.v. injection of Uthol, $1 \mathrm{~mL} / \mathrm{kg}$. The experimental protocol was approved by the Institutional Animal Care and Use Committee of Childrens Hospital Research Foundation (protocol 01093AR).

\section{Blood-Perfused Gut Loop Experiments}

Preparation. Study subjects were anesthetized, intubated, and ventilated to maintain normal blood gas tensions. Heparin $(500 \mathrm{U} / \mathrm{kg})$ was administered. A segment of proximal ileumdistal jejunum $\sim 25 \mathrm{~cm}$ in length was isolated from the remainder of the gut. This segment was cleansed by luminal instillation of warm $\left(38^{\circ} \mathrm{C}\right)$ saline, followed by air. The single arteryvein pair serving this segment was cannulated, and these catheters were attached to a blood-primed extracorporeal perfusion circuit. The segment was removed from the study subject, weighed, and placed into a humidified plexiglass chamber. Tissue temperature was maintained at $38^{\circ} \mathrm{C}$ by means of an overhead heating element connected by a servomechanism to a luminal temperature probe. Arterial perfusion was achieved by an extracorporeal circuit consisting of a collecting flask, arterial and venous reservoirs, and a membrane lung (Sci-Med $0.6 \mathrm{~m}^{2}$ ). Blood for this perfusion circuit was obtained from a second animal approximately $120 \mathrm{~d}$ old $(\sim 35 \mathrm{~kg})$. To this end, blood donor subjects were anesthetized, intubated, ventilated and heparinized as described for experimental subjects; thereafter, $\sim 1000 \mathrm{~mL}$ whole blood was removed while simultaneously infusing swine plasma, obtained during a previous study, at a rate equal to blood withdrawal. Thereafter the donor subject was euthanized. The withdrawn blood was filtered $(40 \mu \mathrm{m})$ and placed in the collecting flask, which in turn was placed in a water bath $\left(39^{\circ} \mathrm{C}\right)$ situated over a stirplate. Blood was continuously circulated $(200 \mathrm{~mL} / \mathrm{min})$ between the collecting flask and the membrane oxygenator, which was gassed with $21 \% \mathrm{O}_{2}-5 \% \mathrm{CO}_{2}$, balance $\mathrm{N}_{2}$. This circulation maintained blood gas tensions within the collecting flask at 12.0-16.0 and 4.5-6.0 kPa for $\mathrm{Po}_{2}$ and $\mathrm{PCO}_{2}$, respectively. A small volume of blood $(\sim 20 \mathrm{~mL})$ was continuously pumped from the collecting flask to an arterial reservoir, which was also placed in the water bath. Flow from the arterial reservoir to the gut loop could be achieved by two means: by pressurizing the reservoir (controlled-pressure perfusion) or by directing the arterial circuit through a Gilson Miniplus 4 pump (controlled flow perfusion). The venous effluent from the gut loop was collected in the venous reservoir and thereafter returned to the collecting flask for recirculation. Gut blood flow was measured by an electromagnetic flowmeter placed in the venous circuit. Standard pressure transducers were connected to the arterial and venous circuits by T-connectors to measure vascular pressures. Blood gas tension and oxygen content determinations were made on samples withdrawn from the arterial and venous circuits by means of a Corning Analyzer and Lex- $\mathrm{O}_{2}-\mathrm{Con}$, respectively.

Protocols. The in vitro gut loop was initially perfused under controlled pressure conditions with the arterial pressure set at $70 \mathrm{~mm} \mathrm{Hg}$ to duplicate in vivo conditions. After the preparation had reached steady state (generally $\sim 30 \mathrm{~min}$ ), baseline measurements were taken. Thereafter, perfusion was switched from controlled pressure to controlled flow by directing the arterial circuit through a pump, and the pump speed was initially set to duplicate the preexisting flow rate. Shortly thereafter, the pump speed was decreased so that gut flow rate was $\sim 40 \%$ of the baseline flow rate. This reduced flow rate was maintained until pressure attained a new steady state value, which generally took $\sim 5$ min. Repeat measurements were taken and the flow was increased by $\sim 30 \%$. This process was repeated until measurements had been taken at six flow rates which ranged from $\sim 40 \%$ to $\sim 170 \%$ of baseline. This protocol was conducted on two groups of study subjects. In the first group, the flow ramp was carried out as described, and then flow returned to baseline. Thereafter, an infusion of LNMMA was begun into the arterial circuit at a rate sufficient to provide an arterial concentration of $0.025 \mathrm{mg} / \mathrm{mL}\left(10^{-4} \mathrm{M}\right)$. This LNMMA dose was chosen as it has been previously determined to fully block NO production by newborn swine mesenteric artery (7). Once hemodynamic variables had reached a new steady state values the flow ramp repeated. The rate of LNMMA infusion was adjusted with each flow change so that the arterial concentration of the drug remained nearly constant. The second group of study subjects underwent similar manipulation except that phenylephrine was infused instead of LNMMA. The arterial concentration of phenylephrine was maintained at 0.0002 $\mathrm{mg} / \mathrm{mL}\left(10^{-6} \mathrm{M}\right)$ during the flow ramp.

\section{Buffer-Perfused Mesenteric Artery Experiments}

Preparation. Subjects were anesthetized and thereafter exsanguinated. The entire mesenteric artery and a small segment of carotid artery were removed and immersed in iced Krebs 
buffer. A 3-4-cm segment of the mesenteric artery was dissected free of neural, lymphatic, and connective tissues, and all branch points were ligated with silk ties. The mesenteric artery segment was mounted between inflow and outflow ports in a water-jacketed glass chamber filled with Krebs buffer (Fig. 1). Perfusion of this segment was achieved with Krebs buffer pumped from a water-jacketed buffer reservoir. An electromagnetic flowmeter and standard pressure transducer within the arterial circuit facilitated measurement of perfusion rate and pressure, respectively. Vasoactive agents released from the mesenteric artery segment were detected by means of bioassay, using a precontracted ring of carotid artery as the bioassay tissue. To this end, the effluent from the mesenteric artery segment could be directly suffused onto the bioassay vessel (transit time $<1 \mathrm{~s}$ ). To keep the rate of effluent delivery to the bioassay vessel constant during manipulation of mesenteric artery flow rate, a second pump (Fig. 1, P2) was added to the effluent arm of the circuit. This pump was controlled by the same potentiometer as that regulating mesenteric artery perfusion; thus, an increase in the perfusion rate resulted in an equal increase in the rate of effluent diversion, so that delivery of the effluent to the bioassay vessel remained constant at $3 \mathrm{~mL} / \mathrm{min}$. The carotid artery bioassay vessel was de-endothelialized by luminal passage of a silk tie. The bioassay vessel was mounted between two wire stirrups, one fixed and the other tethered to a Grass FT03 force transducer. This vessel was stretched to its optimal length over a period of $1 \mathrm{~h}$ as determined by maximal contraction to $20 \mathrm{mM} \mathrm{KCl}$; thereafter, endothelial removal was confirmed by noting $>50 \%$ reduction in tension after application of acetylcholine $\left(10^{-7} \mathrm{M}\right)$. During preparation of the bioassay vessel (i.e. mounting and stretching), its suffusion was derived from a separate water-jacketed buffer reservoir. The bioassay vessel could be moved between this suffusion source and the mesenteric artery effluent by adjusting a micrometer on

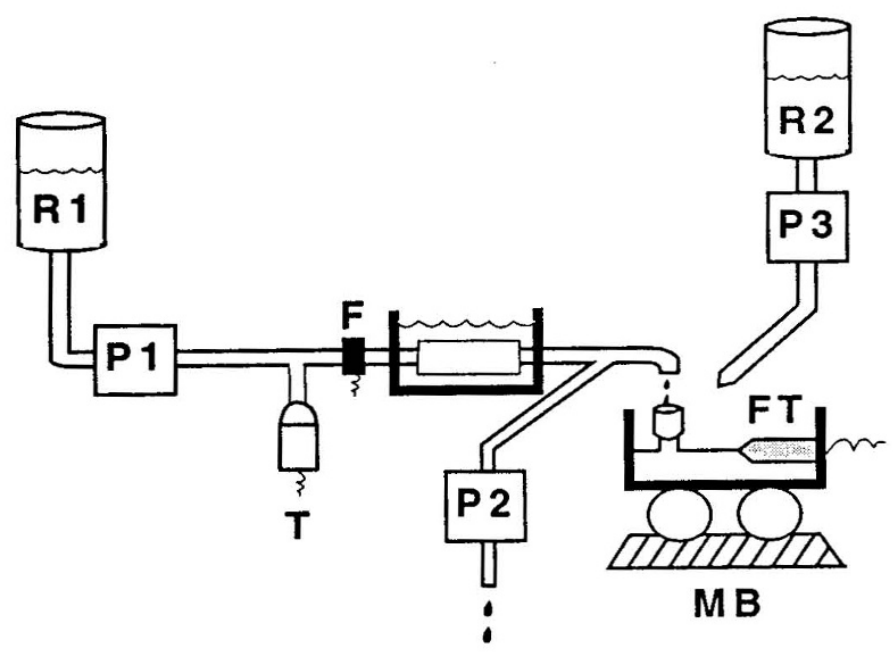

Figure 1. The experimental apparatus used to perfuse in vitro mesenteric artery segments with Krebs buffer. $R$, buffer reservoir; $P$, pump; $T$, pressure transducer; $F$, flow probe; $F T$, force transducer; $M B$, movable base. Pump 1 provides luminal perfusion of the mesenteric artery segment, whereas pump 2 draws off a portion of the effluent, so the that rate of suffusion delivered to the carotid artery bioassay vessel remains constant. The bioassay vessel can be moved so that its suffusion is derived from the mesenteric artery effluent (as pictured) or from a second buffer reservoir, $R 2$. the base of the apparatus. This movement could be carried out without disturbing bioassay vessel tension or force transducer calibration. All buffers were kept at $38^{\circ} \mathrm{C}$ by running warm water through the water-jacketing system that surrounded all reservoirs and chambers. The buffer reservoirs and the mesenteric artery chamber were continuously aerated with $95 \%$ $\mathrm{O}_{2}-5 \% \mathrm{CO}_{2}$

Protocol. After mounting, the mesenteric artery segment was perfused at $10 \mathrm{~mL} / \mathrm{min}$ for $90 \mathrm{~min}$. During this time the effluent from the mesenteric artery was diverted away from the bioassay vessel, so that the latter vessel could be prepared (i.e. stretched). Phenylephrine was then added to both buffer reservoirs (Fig. 1, $R l$ and $R 2$ ) to a final concentration of 0.0002 $\mathrm{mg} / \mathrm{mL}\left(10^{-6} \mathrm{M}\right)$. This action increased tension of the bioassay vessel (i.e. precontraction). Thereafter, the bioassay vessel position was adjusted so that it was suffused by the effluent from the mesenteric artery segment. This circumstance was maintained until the bioassay vessel achieved a new steady state tension (generally $\sim 3 \mathrm{~min}$ ). Thereafter, the rate of mesenteric artery perfusion was increased to $25 \mathrm{~mL} / \mathrm{min}$ and the effect of this perturbation on bioassay vessel tension noted. This protocol was carried out on three groups of study subjects. Group 1 (control) were treated as just described. Indomethacin $\left(10^{-5} \mathrm{M}\right)$ was added to all buffers used in group 2 ; this dose was chosen as it has been previously determined to fully block cyclooxygenase activity in newborn swine mesenteric artery (7). LNMMA $\left(10^{-4} \mathrm{M}\right)$ was added to all buffers used in group 3.

\section{Reagents and Buffers}

Krebs buffer of the following composition was used (in $m M): ~ \mathrm{NaCl}$ 118.06: $\mathrm{KCl} 4.76 ; \mathrm{NaHCO}_{3}$ 24.97; $\mathrm{CaCl}_{2} 2.52$; $\mathrm{MgSO}_{4} 1.22 ; \mathrm{KH}_{2} \mathrm{PO}_{4} 1.19$; disodium EDTA 0.026; dextrose 11.09. This buffer had a $\mathrm{pH}$ of 7.4 when aerated with $95 \%$ $\mathrm{O}_{2}-5 \% \mathrm{CO}_{2}$. LNMMA was obtained from Calbiochem and prepared fresh on the day of use. Phenylephrine and indomethacin were obtained from Sigma Chemical Co. Indomethacin was initially dissolved in an equimolar solution of $\mathrm{NaHCO}_{3}$ and then diluted with saline.

\section{Statistical Methods}

The principal endpoints for the blood perfusion studies were arterial pressure and tissue oxygen uptake, each evaluated as a function of flow rate, and two methods were used to analyze the data. First, data were subjected to a 2-way ANOVA for repetitive measures which used condition (control versus drug treatment, i.e. LNMMA or phenylephrine) and time (i.e. flow rate) as main effects. A significant $f$ statistic indicated that the effect of flow rate on the dependent variable was contingent upon experimental conditions and was followed by post hoc $t$ tests with the Boneferroni correction to determine the sites of difference between conditions. Second, regression analysis was carried out on flow rate versus resistance and oxygen uptake for data within each condition (i.e. control, LNMMA, phenylephrine) to determine the relationships among these variables. These regression analyses were carried out on data collected at all flow rates, and also separately on data collected 
at flows above and below the baseline flow rate. These separate regression equations were compared within and between conditions by ANOVA. The principal endpoint for the buffer perfusion study was relaxation of the bioassay vessel. To this end, relaxation was expressed as a percent change from precontraction tension. These data were subjected to a 2-way ANOVA which used condition (control versus drug i.e., LNMMA or indomethacin) and flow rate as main effects. Post hoc tests were carried out as just described.

\section{RESULTS}

Baseline hemodynamic data are shown in Tables 1 and 2 . Conversion from controlled pressure to controlled flow perfusion caused a modest increase in vascular resistance which did not reach statistical significance. No other measured variables were affected by the change in perfusion mode. Infusion of LNMMA $\left(10^{-4} \mathrm{M}\right)$ at the baseline flow rate increased vascular resistance $27 \pm 5 \%$ but had no effect on tissue oxygenation. Infusion of phenylephrine $\left(10^{-6} \mathrm{M}\right)$ at the baseline flow rate increased vascular resistance $15 \pm 4 \%$ and depressed tissue oxygen uptake $13 \pm 4 \%$. This latter effect was secondary to a phenylephrine-induced reduction in oxygen extraction by the gut, which was most likely consequent to closure of precapillary sphincters, an established effect the $\alpha_{1}$-agonist. The hematocrit of blood within the extracorporeal circuit averaged 28 $\pm 1 \%$, whereas the arterial blood gas and oxygen content values averaged $\mathrm{pH} 7.39 \pm 0.03, \mathrm{Po}_{2} 13.9 \pm 0.8 \mathrm{kPa}, \mathrm{PCO}_{2}$ $5.1 \pm .8 \mathrm{kPa}$, and $4.6 \pm 0.1 \mathrm{mM} \mathrm{O}_{2} / \mathrm{L}$, respectively. These values did not significantly change during the course of the experiments.

In all instances, vascular resistance reached a new steady state value within $1 \mathrm{~min}$ of a step increase in flow rate; no difference in lag time was noted for flows above or below the baseline flow rate. An inverse relationship between flow rate and vascular resistance was noted under control conditions in both LNMMA and phenylephrine groups (Figs. 2 and 3). Thus, reduction of flow to $\sim 40 \%$ of baseline resulted in a significant rise in vascular resistance, whereas elevation of flow to $\sim 170 \%$ of baseline caused a significant reduction in gut vascular resistance. The relationship between these variables could also be appreciated by regression analysis: for control data from the LNMMA group, resistance $=1.08-$ $0.001\{$ flow $\}, r^{2}=0.44, p=0.0069$; for control data from the phenylephrine group, resistance $=1.06-0.002$ flow $\}, r^{2}=$ $0.68, p<0.0001)$. The relationship between flow and tissue oxygen uptake was also similar in both groups: oxygen uptake was independent of flow rate until flow was reduced below $75 \%$ of the baseline flow rate.

Administration of LNMMA $\left(10^{-4} \mathrm{M}\right)$ caused significant vasoconstriction of the intestinal vasculature at all flow rates, although the effect was qualitatively and quantitatively more dramatic at flow rates above baseline. Thus, increasing pump speed above baseline during LNMMA infusion caused significant vasoconstriction in place of the vasodilation noted under control conditions (Fig. 2). The importance of flow rate on LNMMA effect was also confirmed by conducting separate regressions of resistance versus flow rate for data collected at flow rates above and below baseline. For data collected at flows below baseline, the slopes of the regression lines for LNMMA and control data were similar: for LNMMA data, resistance $=1.40-0.004\{$ flow $\}, r^{2}=0.94, p<0.0001$; for control data from the LNMMA group, resistance $=1.25-$ $0.005\{$ flow $\}, r^{2}=0.65, p=0.0025$. For data collected at flows above baseline, the slopes of the regression lines for LNMMA and control data were significantly different: for LNMMA data, resistance $=0.004\{$ flow $\}+0.5, r^{2}=0.62, p=.0008$; for control data from the LNMMA group, resistance $=1.55-$ $0.005\{$ flow $\}, r^{2}=0.58, p=0.0059$. Thus, LNMMA caused a simple vertical shift of the resistance-flow rate curve upward at flows below the baseline flow rate, whereas this agent significantly altered the relationship between these variables at flows above the baseline flow rate. LNMMA had no effect on the relationship between flow rate and intestinal oxygen uptake (Fig. 4).

Phenylephrine $\left(10^{-6} \mathrm{M}\right)$ also caused significant intestinal vasoconstriction, but its effect was not contingent upon flow rate. Instead, this agent induced a simple vertical shift of the resistance-flow rate curve upward throughout the entire range of flows examined (Fig. 3). This effect was also evident by the similarity of the regression lines which describe the relationship between resistance and flow for phenylephrine and control data: for phenylephrine data, resistance $=1.33-0.003\{$ flow $\}$, $r^{2}=0.61, p<0.0001$; for control data from the phenylephrine group, resistance $=1.06-0.002\{$ flow $\}, r^{2}=0.68, p<$ 0.0001 . Separate regression analysis on data collected above and below the baseline flow rate demonstrated similar slopes for all equations. Unlike LNMMA, phenylephrine caused a downward shift in the flow rate-oxygen uptake curve at all flow rates; however, the qualitative relationship between these variables was not affected by phenylephrine (Fig. 5).

Table 1. Hemodynamic and oxygenation data before and during LNMMA infusion within in vitro gut loops from 3-d-old swine, perfused under controlled pressure conditions $(C P)$ or controlled flow $(C F)$ conditions

\begin{tabular}{|c|c|c|c|c|}
\hline & \multicolumn{2}{|c|}{ Control } & \multicolumn{2}{|c|}{ LNMMA } \\
\hline & $\mathrm{CP}$ & $\mathrm{CF}$ & $\mathrm{CP}$ & $\mathrm{CF}$ \\
\hline Arterial pressure $(\mathrm{mm} \mathrm{Hg})$ & $70 \pm 1$ & $75 \pm 2$ & $94 \pm 2^{*}$ & $97 \pm 3^{*}$ \\
\hline Blood flow $(\mathrm{mL} / \mathrm{min} / 100 \mathrm{~g})$ & $101 \pm 3$ & $100 \pm 2$ & $100 \pm 3$ & $100 \pm 3$ \\
\hline Resistance $(\mathrm{mm} \mathrm{Hg} / \mathrm{mL} / \mathrm{min} / 100 \mathrm{~g}$ ) & $0.71 \pm 0.04$ & $0.75 \pm 0.04$ & $0.95 \pm 0.07 *$ & $0.97 \pm 0.06^{*}$ \\
\hline$(a-v) \mathrm{O}_{2}\left(\mathrm{mM} \mathrm{O}_{2} / \mathrm{L}\right)$ & $1.14 \pm 0.06$ & $1.14 \pm 0.06$ & $1.14 \pm 0.11$ & $1.14 \pm 0.07$ \\
\hline Oxygen uptake $\left(\mathrm{mM} \mathrm{O}_{2} / \mathrm{min} / 100 \mathrm{~g}\right)$ & $11.33 \pm 0.32$ & $11.39 \pm 0.29$ & $11.23 \pm 0.25$ & $11.22 \pm 0.21$ \\
\hline
\end{tabular}

Mean \pm SEM, $n=6$ for all observations.

${ }^{*} p<0.01$ vs control. 
Table 2. Hemodynamic and oxygenation data before and during phenylephrine infusion within in vitro gut loops from 3-d-old swine, perfused under controlled pressure conditions $(C P)$ or controlled flow $(C F)$ conditions

\begin{tabular}{|c|c|c|c|c|}
\hline \multirow[b]{2}{*}{$\mathrm{CP}$} & \multicolumn{2}{|c|}{ Control } & \multicolumn{2}{|c|}{ Phenylephrine } \\
\hline & $\mathrm{CF}$ & $\mathrm{CP}$ & $\mathrm{CF}$ & $\mathrm{CF}$ \\
\hline Arterial pressure $(\mathrm{mm} \mathrm{Hg}$ ) & $71 \pm 1$ & $75 \pm 1$ & $95 \pm 2^{*}$ & $98 \pm 2^{*}$ \\
\hline Blood flow $(\mathrm{mL} / \mathrm{min} / 100 \mathrm{~g})$ & $100 \pm 2$ & $100 \pm 3$ & $101 \pm 2$ & $100 \pm 3$ \\
\hline Resistance $(\mathrm{mm} \mathrm{Hg} / \mathrm{mL} / \mathrm{min} / 100 \mathrm{~g})$ & $0.70 \pm 0.06$ & $0.76 \pm 0.04$ & $0.95 \pm 0.06^{*}$ & $0.98 \pm 0.07 *$ \\
\hline$(\mathrm{a}-\mathrm{v}) \mathrm{O}_{2}\left(\mathrm{mM} \mathrm{O}_{2} / \mathrm{L}\right)$ & $1.14 \pm 0.06$ & $1.05 \pm 0.06$ & $0.96 \pm 0.04 *$ & $0.94 \pm 0.06^{*}$ \\
\hline Oxygen uptake $\left(\mathrm{mM} \mathrm{O}_{2} / \mathrm{min} / 100 \mathrm{~g}\right)$ & $11.31 \pm 0.28$ & $11.65 \pm 0.40$ & $10.01 \pm 0.26^{*}$ & $9.56 \pm 0.18 *$ \\
\hline
\end{tabular}

Mean \pm SEM, $n=5$ for all observations.

${ }^{*} p<0.01$ vs control.

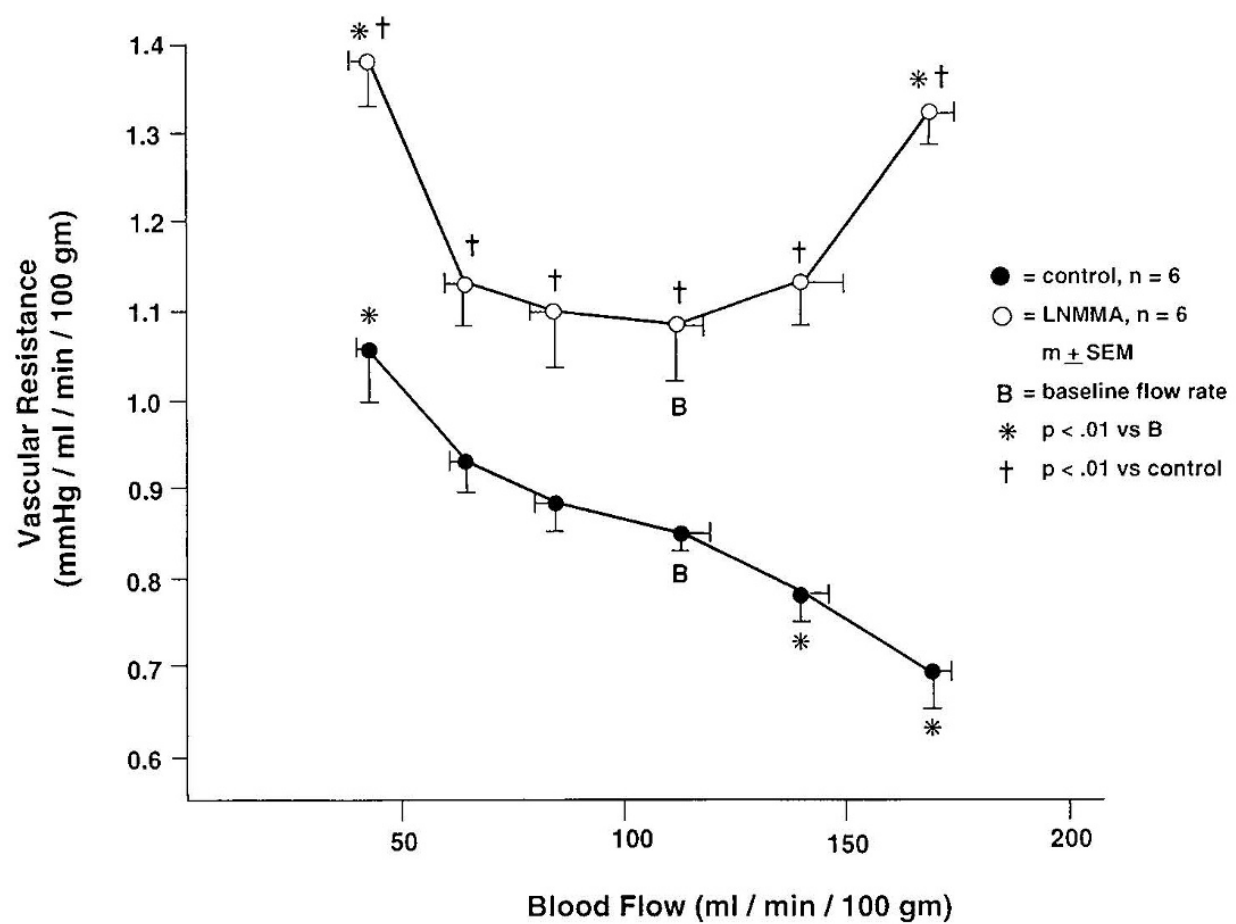

Figure 2. The relationship between blood flow and vascular resistance in blood-perfused in vitro gut loops from 3-d-old swine before and during LNMMA infusion $\left(10^{-4} \mathrm{M}\right)$. Control data are shown as closed circles, whereas data collected during LNMMA infusion are shown as open circles. The $f$ statistic for the 2-way ANOVA was $p=0.0213$. Post hoc $t$ tests were carried out within control and LNMMA data sets against baseline $(B)$, and also between control and LNMMA data sets at each flow rate. Data are given as mean $\pm \mathrm{SEM}, n=6$ for all observations, ${ }^{*} p<0.01 v s B ; p<0.01 v s$ control.

A representative tracing from a buffer-perfused mesenteric artery experiment is shown in Figure 6. A slight reduction in tension of the precontracted bioassay vessel was noted when suffusion with effluent from the mesenteric artery was initiated. Thereafter, increasing the luminal perfusion rate from 10 to 25 $\mathrm{mL} / \mathrm{min}$ caused a significant reduction in bioassay vessel tone. Note that the pressure within the perfusion circuit remained very low during the entire experiment and that pressure did not significantly rise when perfusion flow rate was increased. This stability occurred because no obstruction to buffer outflow was offered in the effluent arm of the perfusion circuit. Combined data are shown in Figure 7. Significant relaxation of the bioassay ring was noted when flow rate was increased from 10 to $25 \mathrm{~mL} / \mathrm{min}$ in control segments. Addition of indomethacin $\left(10^{-5} \mathrm{M}\right)$ had no effect on this response. However, addition of LNMMA $\left(10^{-4} \mathrm{M}\right)$ significantly attenuated dilation of the bioassay ring.

\section{DISCUSSION}

Flow-mediated dilation was clearly evident within the intestinal circulation of 3-d-old swine as vascular resistance decreased with each step increase in flow rate. This phenomenon was noted throughout the entire range of flows studied, a range which encompassed flows well below and above the baseline flow rate characteristic of newborn swine intestine. It was equally clear that the mechanism responsible for flow-mediated dilation was different at high and low flow rates, inasmuch as the qualitative effect of NO synthesis blockade was quite different above and below the baseline flow rate.

Vasodilation noted in response to increments of flow above the baseline flow rate were most likely mediated by NO inasmuch as infusion of an arginine analogue replaced dilation with significant vasoconstriction. The blocking agent used, LNMMA, exerts its vascular effect by disruption of NO synthesis, as evidenced by the reversal of this effect by co-infusion 


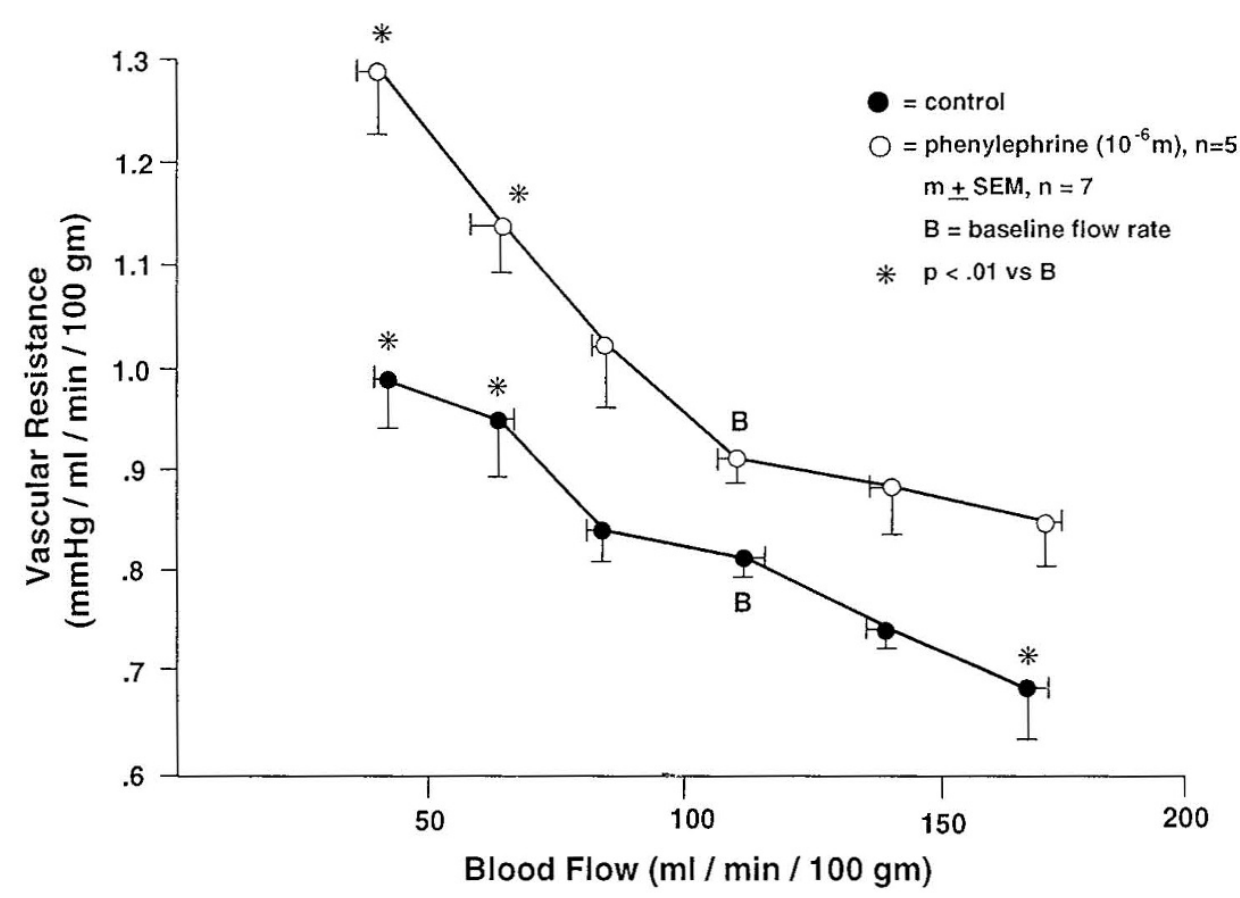

Figure 3. The relationship between blood flow and vascular resistance in blood perfused in vitro gut loops from 3-d-old swine before and during phenylephrine infusion $\left(10^{-6} \mathrm{M}\right)$. Control data are shown as closed circles, whereas data collected during phenylephrine infusion are shown as open circles. The $f$ statistic for the 2-way ANOVA was not significant $(p=0.2756)$, so that the effect of flow rate on vascular resistance was not contingent on phenylephrine infusion. However, regression analysis of resistance $v s$ flow rate was highly significant for both control and phenylephrine data sets (see text); furthermore, the within group main effects for flow rate from the 2-way ANOVA were also highly significant $(p<0.0001)$. Simple 1-way ANOVA were therefore conducted separately on control and phenylephrine data sets to determine which data points were different from baseline; these points are delineated by an asterisk.

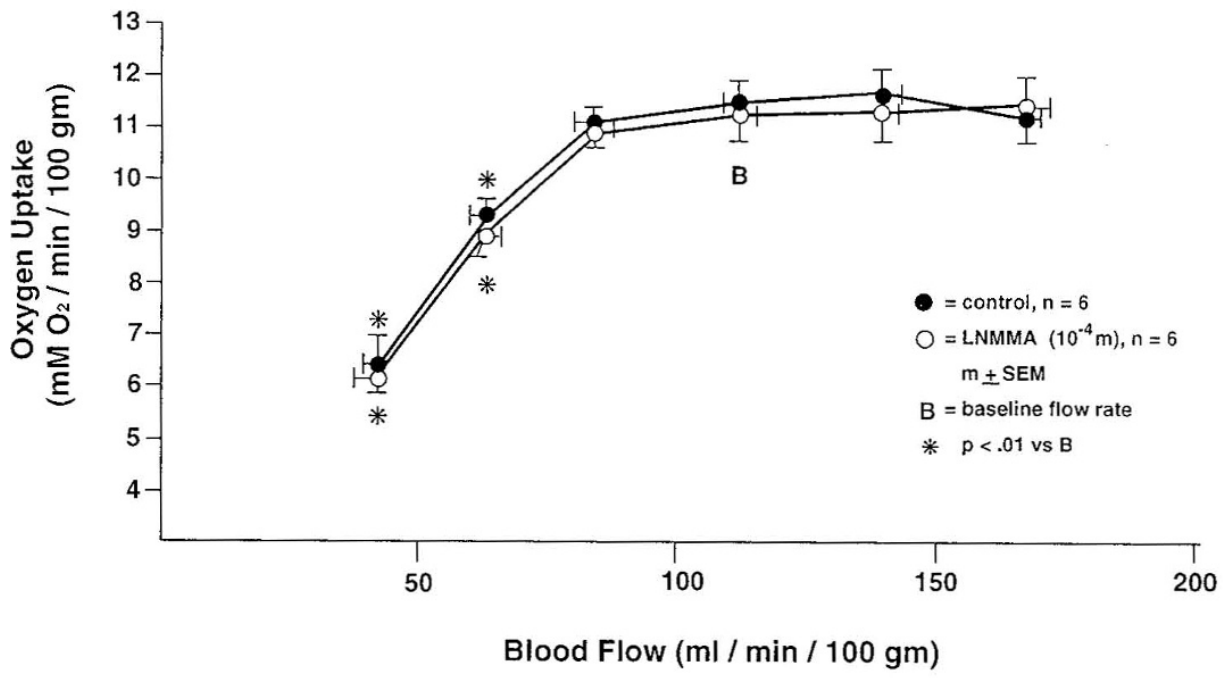

Figure 4. The relationship between blood flow and oxygen uptake in blood-perfused in vitro gut loops from 3-d-old swine before and during LNMMA infusion $\left(10^{-4} \mathrm{M}\right)$. Control data are shown as open circles, whereas data collected during LNMMA infusion are shown as closed circles. The $f$ statistic for the 2-way ANOVA was not significant $(p=0.6604)$, so that the effect of flow rate on oxygen uptake was not contingent upon the presence of LNMMA. However, the within group main effects for flow rate from the 2-way ANOVA were highly significant $(p<0.0001)$. Simple 1-way ANOVA were therefore conducted separately on control and LNMMA data sets to determine which data points were different from baseline; these points are delineated by an asterisk.

of L-arginine $(9,10)$. It might be argued that the effect of LNMMA was also contingent, in part, on the generalized increase in vascular tone that this agent induced, inasmuch as the interaction between flow and pressure can be dependent on the basal tone of the vessel (11). This potential confounding variable was clarified by the phenylephrine studies. Phenylephrine increased baseline vascular tone to a similar degree as LNMMA, but, unlike LNMMA, its effect on resistance was not dependent on flow rate. Phenylephrine is a pure $\alpha_{1}$-agonist which has no effect on NO synthesis or half-life at the concentration applied in this experiment (9). Thus, the effect of LNMMA at flows above the baseline rate were contingent upon its effect on NO production, not on its effect on vascular tone per se. The potential role of NO was also confirmed by the bioassay experiments. Mesenteric artery segments released a transmissible vasodilator in response to a $150 \%$ increase in 


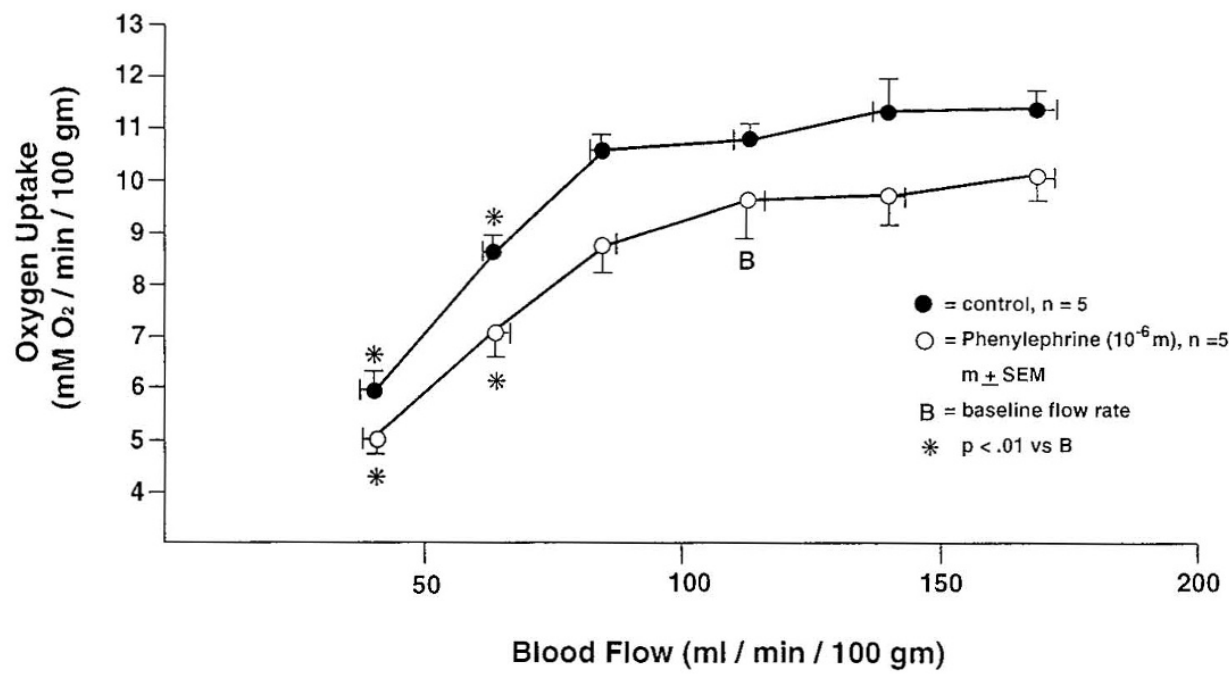

Figure 5. The relationship between blood flow and oxygen uptake in blood-perfused in vitro gut loops from 3-d-old swine before and during phenylephrine infusion $\left(10^{-6} \mathrm{M}\right)$. Control data are shown as open circles, whereas data collected during phenylephrine infusion are shown as closed circles. The $f$ statistic for the 2-way ANOVA was not significant $(p=0.0904)$, so that the effect of flow rate on oxygen uptake was not contingent upon the presence of phenylephrine. However, the within group main effects for flow rate from the 2-way ANOVA were highly significant $(p<0.0001)$. Simple 1-way ANOVA were therefore conducted separately on control and phenylephrine data sets to determine which data points were different from baseline; these points are delineated by an asterisk.
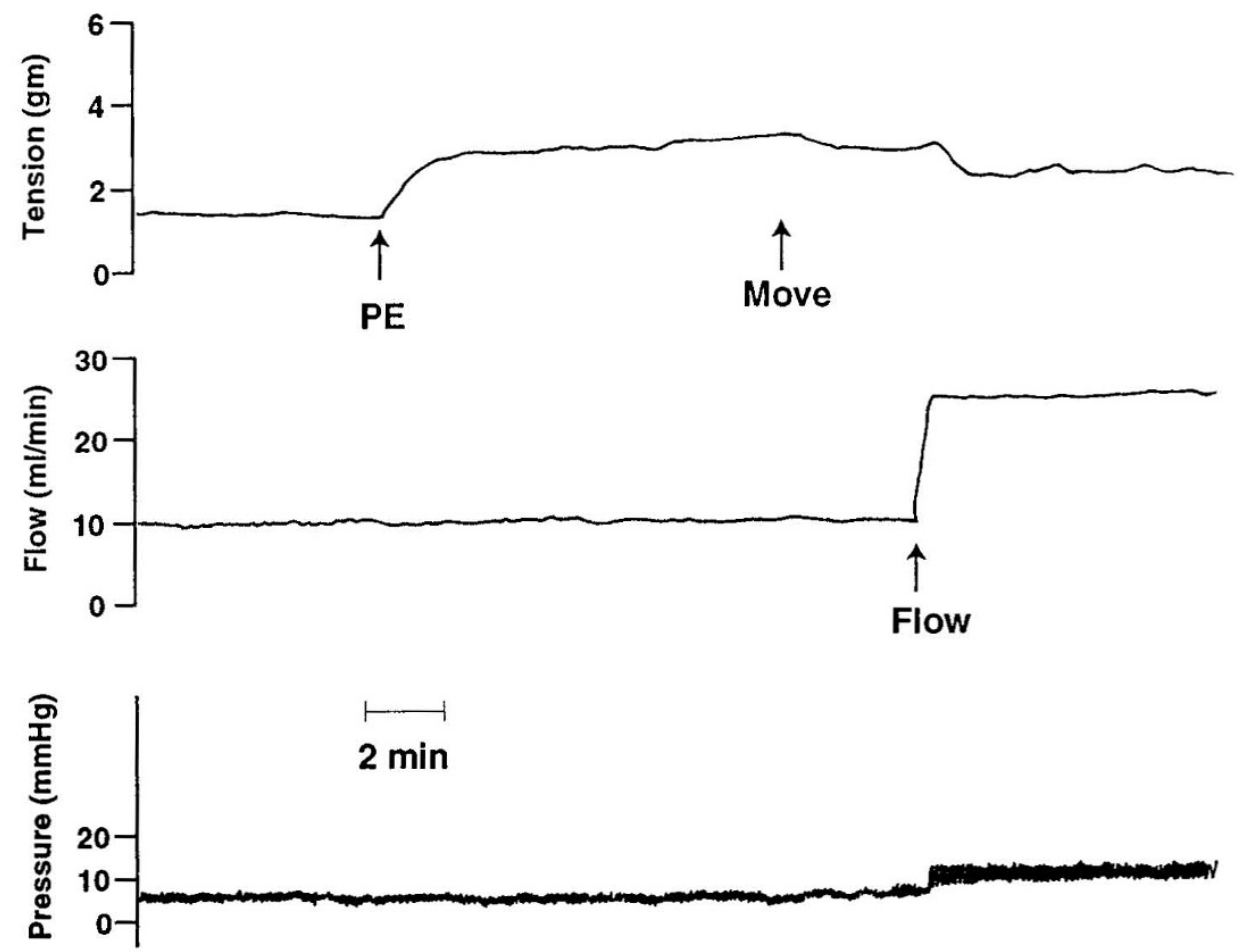

Figure 6. A duplication of a portion of the strip chart recording from a buffer-perfused mesenteric artery experiment. Phenylephrine was added to the buffers at the site marked $P E$; the bioassay vessel was moved into the mesenteric artery effluent at the site marked move; luminal perfusion rate delivered to the mesenteric artery segment was increased from 10 to $25 \mathrm{~mL} / \mathrm{min}$ at the site marked flow.

flow rate; furthermore, this effect was blocked by LNMMA, but not by indomethacin. Although most reports of flowinduced dilation suggest mediation by NO $(3,12,13)$, some have reported a role for endothelium-derived eicosanoids (4). Cyclooxygenase products did not appear to mediate this phenomenon in newborn intestine.

The mechanism(s) responsible for dilation in response to step-increases in flow at rates below the baseline flow rate are less obvious. Neither NO production or resting vascular tone played a significant role in this process as evidenced by the effects of LNMMA or phenylephrine; thus, these agents caused an upward shift of the resistance flow rate curve, without altering the qualitative relationship between these variables. What other factors might have been operative? It is difficult to implicate intrinsic metabolic vascular regulation in this process. The metabolic theory of local vascular control predicts 


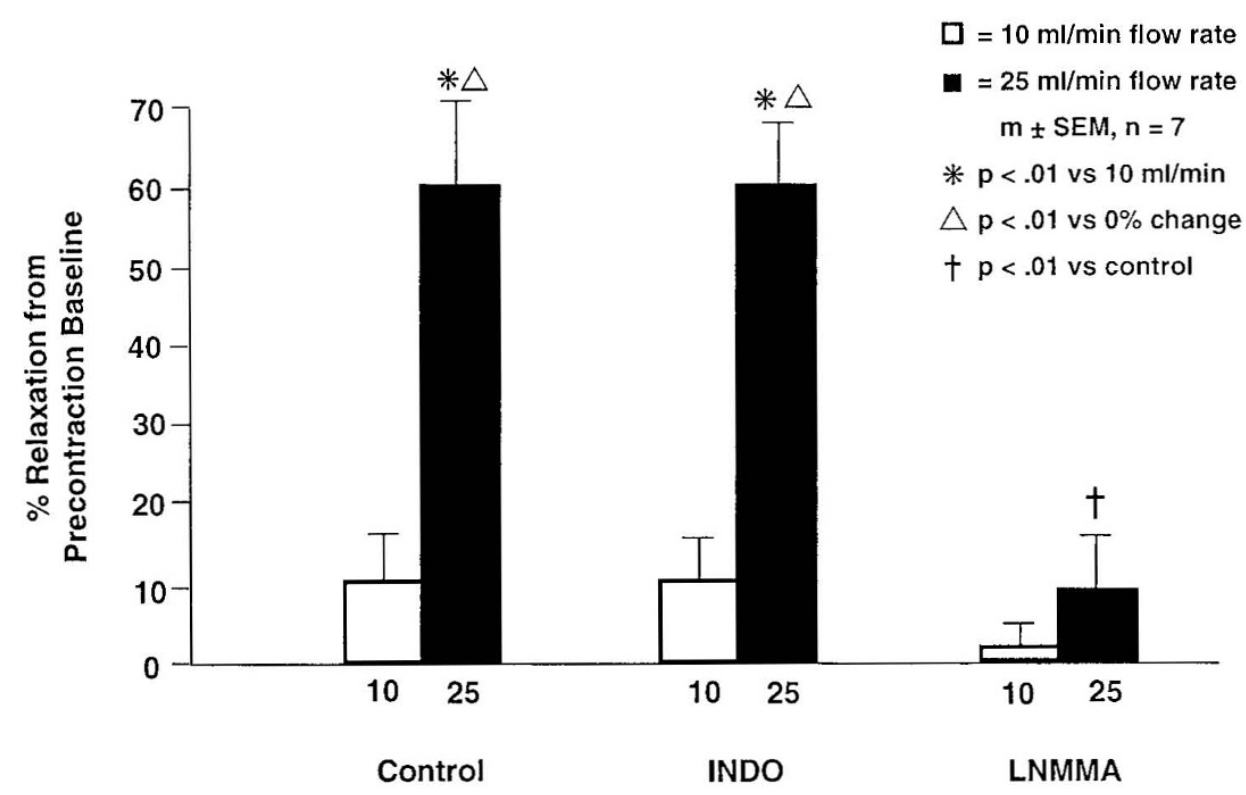

Figure 7. The effect of luminal perfusion rate delivered to the mesenteric artery segment on tension in the phenylephrine-precontracted, deendothelialized carotid artery ring (bioassay vessel). Data are expressed as percent relaxation from precontraction tension. Mean $\pm \mathrm{SEM}, n=7$ for all observations; ${ }^{*} p<0.01$ vs $10 ; p<0.01$ vs $0 \%$ change; $\uparrow p<0.01$ vs control.

vasodilation in response to flow or pressure reduction when tissue oxygenation is compromised $(14,15)$. Based on the flow-oxygen uptake curves generated herein, the metabolic theory would predict maximal vasodilation when tissue oxygenation was compromised, i.e. at the two lowest flow rates. This predicted pattern is opposite that demonstrated experimentally; thus, the intestine demonstrated progressive vasodilation with each step increase in flow rate, even when these increments had no effect on tissue oxygenation. It is also difficult to implicate intrinsic myogenic vascular regulation. The myogenic theory predicts vasoconstriction in response to an increase in intravascular pressure (16), possibly mediated by stretch-regulated ion channels in vascular smooth muscle (17). Again, this predicted behavior was opposite that noted experimentally: vasodilation, rather than vasoconstriction, was noted as flow rate, and thus intravascular pressures were increased under control conditions. In this context, it is interesting to note that administration of LNMMA may have unmasked a myogenic-based vasoconstriction in response to step-increases rates above baseline, as dilation was replaced by constriction during LNMMA infusion. This balance between myogenic-induced vasoconstriction and NO-mediated, flow-induced vasodilation has been recognized in other circulations (18).

Could reduction in the intensity of intrinsic vascular constrictor mechanisms explain the progressive vasodilation noted after each step-increase in flow at rates below the baseline flow rate? Two candidates for this role are angiotensin and endothelin. Local (i.e. endothelial) production of these peptides has been demonstrated in the mesenteric circulations of other species $(19,20)$. More importantly, endothelial production of angiotensin II and endothelin increases during local ischemia and also when NO production is decreased; furthermore, the constrictor effect of these peptides on vascular smooth muscle is inversely related to the local concentration of NO (20-23). This interaction may serve to quickly and dramatically reduce flow to specific circulations in the presence of systemic cardiovascular instability and so contribute to rapid restoration of cardiac output and systemic perfusion pressure $(23,24)$. Might a similar process occur in newborn intestine? Clearly, the present data offer only the opportunity for speculation. The newborn intestinal vasculature demonstrated very pronounced vasodilation in response to the initial step increases in flow rate, well beyond that which might be anticipated based on passive dilation of a relatively collapsed (i.e., blood volume depleted, ischemic) circulation $(9,10)$. It is feasible that part of this pronounced early vasodilation might represent reduction of constrictor peptide production and action, modulated by the increase in flow rate. This circumstance might explain the relative tendency of the newborn intestinal vasculature to constrict in response a reduction in flow rate or pressure (8), as well as the pronounced vasodilation noted herein during the early step increases in flow rate.

Several limitations to data interpretation deserve mention. First, the phenomenon of flow-induced dilation actually appears to be mediated by the stimulus of wall shear stress $(4,6)$. Shear stress is a tangential force exerted on the vessel wall derived by the friction of flowing blood against the stationary endothelium. In its most simplified version, shear stress is given by the equation: $\tau=4 \mu Q / \pi r^{3}$, where $\tau$ is shear stress, $\mu$ is viscosity, $Q$ is flow rate, and $r$ is vessel radius. Vasodilation increases vessel radius, and so restores shear stress. The experimental design of the present study precluded measurement of vessel radius and so did not allow calculation of wall shear stress. Although it is quite certain that augmentation of flow rate increased shear stress, the actual magnitude of this increase is not known; this deficiency precludes estimation of the gain between stimulus (i.e., shear stress) and response (i.e., dilation). Second, the present data provide no insight as to the site within the intestinal circulation where flow-induced dilation occurs, that is, the macrocirculation within the mesentery 
versus the microcirculation within the wall of the intestine. Such information might suggest possible interactions between flow-induced dilation and other vascular effectors that regulate intestinal vascular tone. Third, the buffer perfusion studies were not carried out in de-endothelialized mesenteric artery segments. Attenuation of bioassay vessel relaxation after treatment with LNMMA strongly suggests a role for NO, which would most likely be endothelium-derived under the present experimental conditions. Absolute confirmation of the endothelial role in this process would require observations within an endothelium-denuded mesenteric artery preparation. Attempts to create such a circumstance proved unsuccessful, possibly because of the multiple ligated branch points present on the mesenteric artery segments. Removal of the endothelium proximal to the ligation ties could not be successfully accomplished without causing excessive damage to the vessel. Finally, it is possible that the bioassay vessel dilation noted after augmentation of flow rate was consequent to a "washout" of dilator from the mesenteric artery. This action seems unlikely, however, for two reasons. First, the bioassay vessel dilation was eliminated by LNMMA, an agent whose sole vascular effect is mediated by its action on NO synthesis $(9,10)$. Second, NO is not stored after its production, but rather is immediately released; furthermore, its half-life is very brief, being measured in seconds $(24,25)$.

The presence of flow-induced dilation in the newborn intestinal circulation may be of physiologic relevance from the perspective of splanchnic oxygen transport. The rate of oxygen uptake by 3 -d-old gut is nearly twice that noted during later postnatal life. To maintain this increased rate of oxygen uptake the newborn intestine relies heavily on a brisk rate of blood flow, and thus oxygen delivery; indeed, the oxygen delivery:uptake ratio is actually higher and (a-v) $\mathrm{O}_{2}$ lower in newborn intestine (8). These circumstances assure a generous capillary $\mathrm{PO}_{2}$ within newborn intestine (8), which in turn has two important consequences: first, it assures an adequate driving force for oxygen diffusion from capillary-to-cell within the intestine (15); second, it facilitates increased oxygen delivery to the liver, inasmuch as portal venous $\mathrm{PO}_{2}$ is relatively high (26). This entire circumstance is contingent upon preservation of a very low vascular resistance in newborn intestine. In this context, the relationship between flow rate and endothelial NO production in newborn intestine might represent a specific vascular adaptation inasmuch as it would participate in maintenance of gut vascular conductance during transition from fetal to neonatal life, a time when the oxygen demand of the intestine is enormous. If this speculation is correct, then factors which disrupt endothelial cell function (e.g. cytokines such as tumor necrosis factor or platelet-activating factor, bacterial toxins, and the ischemia-reperfusion cycle) could significantly impair intestinal perfusion by attenuating an important mechanism of flow control.

Acknowledgment. The authors thank Mary Smith for secretarial support.

\section{REFERENCES}

1. Lie M, Sejersted OM, Kiil F 1970 Local regulation of vascular cross section during changes in femoral artery blood flow in dogs. Circ Res 27:727-737

2. Hull SS, Kaiser L, Jaffe MD, Sparks HV 1986 Endothelium-dependent flow-induced dilation of canine femoral and saphenous arteries. Blood Vessels 23:183-198

3. Smiesko V, Lang DJ, Johnson PC 1989 Dilator responses of rat mesenteric arcading arterioles to increased blood flow velocity. Am J Physiol 257:H1958-H1965

4. Koller A, Sun D, Kaley G 1993 Role of shear stress and endothelial prostaglandins in flow- and viscosity-induced dilation of arterioles in vitro. Circ Res 72:1276-1284

5. Kelm M, Schrader J 1990 Control of coronary vascular tone by nitric oxide. Circ Res 66:1561-1575

6. Lamontagne D, Pohl U, Busse R 1992 Mechanical deformation of vessel wall and shear stress determine the basal release of endothelium-derived relaxing factor in the intact rabbit coronary vascular bed. Circ Res 70:123-130

7. Nowicki PT, Edwards RE 1992 Effect of $N^{G}$-monomethyl-L-arginine on postnatal intestinal vascular resistance is age dependent. Pediatr Res 31:64(abstr)

8. Nowicki PT, Miller CE 1993 Regulation of capillary exchange capacity in postnatal swine intestine. Am J Physiol 265:G1090-G1097

9. Rees DD, Palmer RMJ, Hodson HF, Moncada S 1989 A specific inhibitor of nitric oxide formation from $\mathrm{L}$-arginine attenuated endothelium-dependent relaxation. $\mathrm{Br} \mathrm{J}$ Pharmacol 96:418-424

10. Palmer RMJ, Rees DD, Ashton DS, Moncada S 1988 L-Arginine is the physiological precursor for the formation of nitric oxide in endothelium-dependent relaxation. Biochem Biophys Res Commun 153:1251-1256

11. Johnson PC 1986 Autoregulation of blood flow. Circ Res 59:483-495

12. Hecker M, Mulsch A, Bassenge E, Busse R 1993 Vasoconstriction and increased flow: two principal mechanisms of shear stress-dependent endothelial autocoid release. Am J Physiol 265:H828-H833

13. Cooke JP, Rossitch E, Andon NA, Loscaizo J, Dzau VJ 1991 Flow activates an endothelial potassium channel to release endogenous nitrovasodilator. J Clin Invest $88: 1663-1671$

14. Shepherd AP 1982 Metabolic control of intestinal oxygenation and blood flow. Fed Proc 41:2084-2089

15. Granger HJ, Shepherd AP 1979 Dynamics and control of the microcirculation. Adv Biomed Eng Med Phys 7:1-62

16. Folkow B 1964 Description of the myogenic hypothesis. Circ Res 15:279-287

17. Meininger GA, Davis MJ 1992 Cellular mechanisms involved in the vascular myogenic response. Am J Physiol 263:H647-H659

18. Kuo L, Davis MA, Chilian WM 1990 Endothelium-dependent, flow-induced dilation of isolated coronary arteries. Am J Physiol 259:H1063-H1070

19. Kifor I, Dzau V 1987 Endothelial renin-angiotensin pathway: evidence for intracellular synthesis and secretion of angiotensins. Circ Res 60:422-428

20. Yilmaz G, Aksulu HE, Ercan ZS, Zengil H, Turker RK 1987 Modulation by endothelium of the vascular effects of angiotensin II. Agents Actions 21:184-190

21. Ahokas RA, Sibai BM 1992 Endothelium-derived relaxing factor inhibition augments angiotensin II reactivity in pregnant rat hindlimb. Am J Obstet Gynecol 167:10531058

22. Ito S, Juncos LA, Nushiro N, Johnson C, Carretero OA 1991 Endothelium-derived relaxing factor modulates endothelin action in afferent arterioles. Hypertension 17:1052-1056

23. Lerman A, Sandok E, Hildebrand FF, Burnett JC 1992 Inhibition of EDRF enhances endothelin mediated vasoconstriction. Circulation 85:1894-1898

24. Faraci FM 1990 Role of nitric oxide in regulation of basilar artery tone in vivo. Am J Physiol 259:H1216-H1221

25. Rees DD, Palmer RMJ, Moncada S 1989 Role of endothelium-derived nitric oxide in the regulation of blood pressure. Proc Nat Acad Sci USA 86:3375-3378

26. Edelstone DI, Holzman IR 1981 Oxygen consumption by the gastrointestinal tract and liver in conscious newborn lambs. Am J Physiol 240:G297-G304 